\title{
Reported outcomes for planned caesarean section versus planned vaginal birth in women aged 18-45: a systematic review
}

\author{
Hui Wei Leow ${ }^{1}$, Elizabeth Tan $^{2}$, and Mairead Black ${ }^{3}$ \\ ${ }^{1}$ The University of Edinburgh College of Medicine and Veterinary Medicine \\ ${ }^{2}$ University of Aberdeen \\ ${ }^{3}$ Aberdeen Maternity Hospital
}

May 19, 2020

\begin{abstract}
Background There is currently no consensus regarding the outcomes and outcome measures that should be reported in studies assessing planned mode of birth. Objectives To develop an inventory for reported outcomes for studies comparing planned caesarean section (CS) and planned vaginal delivery (VD) for women age 18-45. Search strategy Three online databases, Ovid SP version of MEDLINE and EMBASE and the Cochrane Pregnancy and Childbirth Group's Trials Register, were searched from 2011 to June 2019. Selection criteria The inclusion criteria were: prospective studies evaluating planned mode of birth, age 18-45, singleton pregnancy, gestational age 37-40 weeks, >100 participants, middle or high income countries. No language restrictions were applied. Data collection and analysis Two researchers independently screened titles and abstracts, and subsequently reviewed the full text of each selected study to assess for eligibility. Discrepancies were resolved by discussion with a third author. The selected studies were evaluated using the MOMENT criteria. Outcomes and outcome measures were systematically extracted and organised into an inventory. Main results 63 prospective studies including data from 6,397,310 women were included. Based on the MOMENT score of 4 or more, 37 studies (59\%) were of high quality. In total, 43 different primary outcomes and 79 different primary outcome measures were identified; 12 different secondary outcomes and 31 secondary outcome measures were identified. The primary outcome measurements and definitions of outcomes were inconsistent. Conclusions Prospective studies evaluating outcomes of planned mode of birth frequently report different primary outcomes and outcome measures. Funding: None required
\end{abstract}

Reported outcomes for planned caesarean section versus planned vaginal birth in women aged 18-45: a systematic review

Authors: Hui Wei Leow ${ }^{1}$, Elizabeth Lilinn Tan $^{2}$, Mairead Black ${ }^{2}$

1. University of Edinburgh, 47 Little France Crescent, Edinburgh EH16 4TJ

2. University of Aberdeen, Polwarth Building, Foresterhill, Aberdeen AB25 2ZD

Contact:

Dr Hui Wei Leow dramandaleow@gmail.com

Ms Elizabeth Lilinn Tan lilinn.tan.16@abdn.ac.uk

Dr Mairead Black mairead.black@abdn.ac.uk

Corresponding author:

Dr Mairead Black

Room 6 Aberdeen Centre for Women's Health Research 
Second floor, Aberdeen Maternity Hospital

Cornhill Road, Aberdeen, AB252ZD

Short running title: Reported outcomes for planned mode of birth

Abstract

Background

There is currently no consensus regarding the outcomes and outcome measures that should be reported in studies assessing planned mode of birth.

\section{Objectives}

To develop an inventory for reported outcomes for studies comparing planned caesarean section (CS) and planned vaginal delivery (VD) for women age 18-45.

\section{Search strategy}

Three online databases, Ovid SP version of MEDLINE and EMBASE and the Cochrane Pregnancy and Childbirth Group's Trials Register, were searched from 2011 to June 2019.

\section{Selection criteria}

The inclusion criteria were: prospective studies evaluating planned mode of birth, age 18-45, singleton pregnancy, gestational age 37-40 weeks, $>100$ participants, middle or high income countries. No language restrictions were applied.

\section{Data collection and analysis}

Two researchers independently screened titles and abstracts, and subsequently reviewed the full text of each selected study to assess for eligibility. Discrepancies were resolved by discussion with a third author. The selected studies were evaluated using the MOMENT criteria. Outcomes and outcome measures were systematically extracted and organised into an inventory.

\section{Main results}

63 prospective studies including data from 6,397,310 women were included. Based on the MOMENT score of 4 or more, 37 studies (59\%) were of high quality. In total, 43 different primary outcomes and 79 different primary outcome measures were identified; 12 different secondary outcomes and 31 secondary outcome measures were identified. The primary outcome measurements and definitions of outcomes were inconsistent.

\section{Conclusions}

Prospective studies evaluating outcomes of planned mode of birth frequently report different primary outcomes and outcome measures.

Funding: None required

Keywords: Caesarean section; vaginal delivery; pregnancy; systematic review; core outcomes; outcome harmonization; outcome variation; COMET; CROWN initiative

(Abstract max 250 words)

PROSPERO registration : CRD42019133104.

\section{Tweetable abstract}

Reported outcomes and outcome measures of planned mode of birth studies are inconsistent and require standardisation. (110 characters max)

\section{Background}


Planned caesarean section (CS) is poorly understood as studies reporting CS outcomes often combine results for emergency and planned CS or compare outcomes with actual vaginal births only. This represents a substantial gap in knowledge as the alternative to planned CS is to plan a vaginal birth. As emergency CS is usually an outcome of planning vaginal birth, it is critical that the outcome of planned and emergency CS are analysed separately. Given that CS rate is rising progressively with reported rates of $24.5 \%$ in Western Europe and $32 \%$ in North America, there is a need for high quality evidence comparing planned CS to planned VD ${ }^{1-4}$.

There is no consensus among patients, healthcare professionals and researchers regarding the outcome measures that should be collected and reported in studies assessing planned mode of birth. In absence of a standardised approach, researchers can only make arbitrary decisions when choosing among several important outcomes ${ }^{5}$. Furthermore, reporting bias can occur if the outcome is selected retrospectively based on statistical significance of results ${ }^{6}$. In order for future studies to be comparable and for results to be usefully combined, it is crucial for reporting of outcomes to be consistent ${ }^{7}$.

Core outcome sets capture the key outcomes based on what key stakeholders (clinicians, patients, their partners, researchers, service developers, funding organisations) consider important in the management or prevention of a condition ${ }^{8,9}$. Ideally, the primary outcome and other outcomes of a trial will be selected from a core outcome set if available, but researchers may continue to explore other outcomes and are not restricted to the outcomes in the core outcome set ${ }^{9}$. The RCOG and Royal Australian and New Zealand College of Obstetricians and Gynaecologists (RANZCOG) has formed the Core Outcomes in Women's and Newborn's Health (CROWN) initiative, which is supported by 80 journals, to tackle unwarranted variation in outcome reporting by encouraging the publication of studies reporting outcomes from a core outcome set where available ${ }^{10,11}$.

For a meaningful core outcome set for planned mode of birth to be developed, evaluation of reported outcomes and outcome measures across published studies is required ${ }^{12}$. The aim of the present study was to identify and organise the outcomes and outcome measures reported in prospective studies investigating planned mode of birth.

\section{Methods}

A protocol including study selection criteria and data extraction methods was developed. The systematic review was registered with the International Prospective Register of Systematic Reviews (PROSPERO) (registration number: CRD42019133104; Available from: https://www.crd.york.ac.uk/prospero/display_record.php?ID=CRD42019133104; Registered on 04 September 2019), and was conducted in accordance with the Preferred Reporting Items for Systematic Reviews and Meta-Analyses (PRISMA) statement ${ }^{13}$. Ethical approval was not required for this study. This study did not require any funding.

\section{Search methods for identification of studies}

The review was undertaken by searching the Ovid SP version of MEDLINE and EMBASE and the Cochrane Pregnancy and Childbirth Group's Trials Register from 2011 to June 2019, followed by a manual search of references of relevant articles (reviews) and clinical guidelines (ACOG, NICE, UpToDate) for eligible studies. The length of the review period was restricted to 2011 to June 2019 because NICE clinical guideline for planned CS was updated in 2011 and scoping work showed that this guideline would enable identification of all relevant studies to capture the most important outcomes up until that date. The search strategy used in electronic databases was based upon a combination of terms relating to caesarean section; vaginal birth; and planned (Appendix S1). Abstracts and conference proceedings were excluded because of the high probability of incomplete data. Citations were collated using EndNote X9 and Microsoft Excel and duplicates were removed.

\section{Study selection}

The inclusion and exclusion criteria of the study are as in Table 1. Only prospective studies reporting clinical outcomes of planned CS versus planned vaginal birth at term for singleton pregnancy were included. 
All cases where vaginal birth was the intended birth route regardless of whether this resulted in emergency caesarean section, forceps delivery, ventouse delivery or spontaneous vaginal birth were included. Studies that classified women with planned VD who eventually had planned VD, and women with planned VD who eventually had emergency CS as separate interventions were included. This is because these studies added value to our overall objective of collecting core outcome and outcome measurements, and our review did not assess results of outcomes between the two different groups of planned VD including emergency birth versus planned CS, where incomplete groups would lead be a major weakness.

Outcomes reported for multiple pregnancy, preterm birth and vaginal birth after CS (VBAC), also known as trial of labour after CS (TOLAC), were not included as these topics would warrant separate core outcome sets ${ }^{14,15}$. Studies investigating placenta praevia, HIV infection, contracted pelvis were excluded as these were clear indications for CS. Abstracts, reviews and conference proceedings were excluded. Retrospective studies were excluded due to high risk of reporting bias. Studies comparing planned mode of birth of second pregnancy versus first pregnancy were excluded as they were not explicitly comparing planned CS versus planned VD. There were no language restrictions.

\section{Data extraction and management}

Two researchers (HWL, ELT) independently screened titles and abstracts, and subsequently reviewed the full text of each selected study to assess for eligibility. Any discrepancies in initial screening between the two researchers were resolved by discussion with a third author (MB). The following data were extracted from the selected studies: participant demographics (sample size, age, geographic), study design, interventions (spontaneous vaginal delivery (SVD), operative VD, planned/ elective CS or unplanned/ emergency CS), primary and secondary outcomes, outcome measures and definitions (if given). If the primary outcome was not stated explicitly, the main outcome reported in the conclusion or the outcome included in the study's power calculation was recorded. The authors of the studies were not contacted to clarify the details of primary outcome. The composite outcomes were recorded in the extraction of outcomes.

\section{Quality assessment}

The outcome quality was scored according to the MOMENT criteria (Management of otitis media with effusion in cleft palate score system) in a 6-point scale. The MOMENT criteria is a validated checklist used for quality assessment at study level which has been employed in a previous core outcome set development for women's health and asks the following ${ }^{16,17}$ : was a primary outcome stated (one point); was the primary outcome clearly defined for reproducible measures (one point); were the secondary outcomes clearly stated (one point); were the secondary outcomes clearly defined for reproducible measures (one point); do the authors explain the choice of outcome (one point); and are the methods used designed to enhance quality of measures appropriate (one point) ${ }^{18}$. High quality was determined for studies that reached score 4 or more in these criteria. Although composite outcomes were recorded, they were not scored as primary outcomes because they are known to be frequently unreasonably combined, inconsistently defined and inadequately reported ${ }^{19}$.

For this review, the studies were not excluded based on their quality as the aim of this review was not to assess efficacy or causal associations of mode of birth on outcomes, but simply to provide a summary of outcomes reported in previous studies, and therefore was not considered to be affected by study quality.

\section{Data analyses}

The identified outcomes were organised into an inventory based on the outcome measures and quality of the studies. The total number of individual outcomes and the frequency of each reported outcome were calculated and definitions of outcomes were analysed. The outcomes were reviewed to reduce duplication caused by different terminologies and similar outcomes were grouped together to create a clear and succinct outcome inventory. A narrative synthesis was performed in addition to the summative analysis. The outcomes were organised into broad categories: maternal and neonatal short-term and long-term outcomes and subsequent pregnancy outcomes. The short-term outcomes were defined as outcomes less than one year after delivery 
and long-term outcomes as one year or more.

\section{Results}

In total, 1878 titles and abstracts were screened, 99 potentially relevant studies were examined in detail (Figure 1). 63 prospective studies (two case-control studies, 48 cohort studies, 7 cross-sectional studies, 6 observational studies) reporting data from 6,397,310 women met the inclusion criteria (Appendix S2). All studies reported outcomes comparing planned mode of birth, 36 studies also reported outcomes for emergency CS (57\%), 17 studies also reported outcomes for assisted VD, 8 studies reported outcomes for planned VD vs CS for breech birth (13\%) (Appendix S2).

Based on the MOMENT score of 4 or more, 37 studies (59\%) were of high quality (Appendix S3). The primary outcome was clearly stated in 50 studies (79\%), 49 studies (78\%) had reproducible primary outcome measures. 11 studies reported secondary outcomes (18\%), 6 (10\%) studies had reproducible secondary outcome measures. In 51 studies (81\%), the authors explained the choice of outcomes selected. 46 studies (73\%) employed methods to enhance quality of measures by suitable training, repetition, or adjusting for confounding factors. 3 studies reported composite outcomes studying childhood adverse neurodevelopmental outcome, neonatal morbidity outcomes, and maternal and neonatal complications (Appendix S3) ${ }^{20-22}$.

In total, 43 different primary outcomes and 79 different primary outcome measures were identified; 12 different secondary outcomes and 31 secondary outcome measures were identified (Table 2). The most reported outcomes were maternal short-term outcomes $(\mathrm{n}=26$ outcomes), followed by childhood long-term outcomes $(n=11$ outcomes), neonatal short-term outcomes $(n=9)$, maternal long-term outcomes $(n=8)$, and subsequent pregnancy outcomes $(\mathrm{n}=1)$ (Table 2$)$.

The primary outcomes and secondary outcomes were organised into an inventory (Table 3; Table S1 and S2). Maternal primary outcomes were organised into broad categories (Table S1): maternal experiences and feelings $(n=11$ studies), genitourinary symptoms $(n=9)$, postpartum complications/ morbidity $(n=9)$, maternal experiences and feelings $(n=11)$, breastfeeding $(n=5)$ and hormone levels $(n=1)$. The most reported primary maternal outcomes were breastfeeding $(n=5$ studies, $8 \%)$ and postpartum depression ( $n=6$ studies, $10 \%$ ). Neonatal outcomes were organised into broad categories: baseline measurements $(\mathrm{n}=10$ studies $)$ and morbidity $(\mathrm{n}=7)$. The most reported outcome measurements were baseline measurements such as SpO2, HR, cord blood $\mathrm{pH}$ after birth and APGAR score (Table S1). Childhood long-term outcomes were categorised into different health conditions (metabolic diseases, $n=4$; respiratory, $n=4$ ) and neurodevelopmental outcomes ( $\mathrm{n}=9$ studies). Two studies looked into maternal subsequent pregnancy outcomes (Table S1).

The secondary maternal outcomes were organised into broad categories (Table S2): genitourinary complications $(n=7$ studies), maternal experiences and feelings $(n=3)$, maternal morbidities $(n=2)$, breastfeeding $(n=2)$ and baseline measurements $(n=1)$. The most reported outcome was anal and urinary incontinence $(n=3)$. Two studies reported secondary neonatal outcomes and one study reported childhood long-term outcomes. Various scales/ questionnaires were used to measure maternal primary outcome measures (n=15 scales/ questionnaires), childhood primary outcome measures $(n=2)$, maternal secondary outcome measures $(n=5)$, childhood secondary outcome measure $(n=1)$ (Table 4$)$.

\section{Discussion}

\section{Main findings}

This systematic review demonstrated a wide variation in reported outcomes and outcome measures in most studies investigating planned mode of birth. 63 prospective studies including data from 6,397,310 women were include. $79 \%$ of the studies reported a primary outcome. Among the reported outcomes, maternal outcomes were the most reported ones (47\%), followed by childhood long-term outcomes (20\%), neonatal short-term outcomes (16.4\%), maternal long-term outcomes (14.6\%) and subsequent pregnancy outcomes $(1.8 \%)$ (Table 2). This variation in outcome reporting could be due to differences in ease of data collection for long-term outcomes compared to short-term outcomes for maternal health, whereas for neonatal outcome, immediate outcomes may be more easily collected than to follow-up with children's long-term health. Only 
one subsequent pregnancy outcome was recorded but this could be due to the nature of this systematic review that only studies comparing planned CS versus planned VD were included rather than studies comparing first and second pregnancy. The variation in outcome reporting and outcome measures means that individual studies cannot be compared and combined in a meta-analysis of outcomes of planned mode of birth, thereby limiting its usefulness to inform clinical practice.

\section{Strengths and Limitations}

To our knowledge, this is the first systematic review to identify key outcomes reported in studies evaluating planned mode of birth. The strengths of the present study include its comprehensive search strategy and methodological design following methodology of previous publications in this field ${ }^{12,23}$. To prevent bias in the review process, study selection was conducted independently by two researchers and discrepancies were resolved through discussion. However, the exclusion of retrospective studies and strict inclusion criteria meant that certain useful reported outcomes may have been omitted. Whilst it may be useful to review retrospective trials, the outcome selections in retrospective trials could be influenced by many factors, including finances and time frame, and tend to have reporting bias, therefore affecting the quality of outcomes and outcome measures selected. The high prevalence of reporting bias could have an impact on the conclusions in Cochrane reviews ${ }^{24}$. Given that up to $85 \%$ of studies are wasted throughout various stages of research, accumulating a loss of tens of billions of dollars of investment in research annually, improved methodological input for research design at an early stage, such as selecting appropriate outcomes, is detrimental ${ }^{25}$.

\section{Interpretation}

The next challenge is to address poor outcome reporting by developing a core outcome set for planned mode of birth. By selecting key outcomes from a core outcome set and using appropriate outcome measures, future studies can reduce inconsistency for outcome reporting, and can thereby improve clinical usefulness of studies ${ }^{8}$. Core outcome sets may be developed with use of the modified Delphi survey where key stakeholders including patients are involved in selecting important reporting outcomes, followed by consensus discussions $^{8,23}$. The Core Outcome Measures in Effectiveness Trials (COMET) initiative advocate for patient and public involvement in the development of core outcome sets because their contribution towards the understanding of important outcomes is essential in the development of patient-centred core outcome sets

9. The development of core outcome sets is supported by Cochrane Reviews of the effects of healthcare interventions and by the World Health Organisation (WHO) in developing guideline recommendations ${ }^{7}$.

Across women's health, several core outcome sets, including pre-eclampsia, endometriosis and infertility, have been developed ${ }^{12}, 23,26,27$. The successful implementation of a core outcome set for rheumatoid arthritis has been shown to improve harmonisation of research by establishing outcomes that are now more frequently measured by researchers ${ }^{24}$.

This study demonstrates that the reporting outcomes for planned mode of birth is inconsistent and requires standardisation. There is no internationally agreed selection of outcomes for studies evaluating planned mode of birth. Future trials should select the primary outcome from the core outcome set alongside other outcomes. This will improve possibility of comparing and combining outcomes from different studies for meta-analyses and will reduce reporting bias ${ }^{28}$.

\section{Conclusion}

In conclusion, there is lack of standardised outcomes and outcome reporting measures among prospective studies evaluating planned mode of birth. The development of a core outcome set for planned mode of birth is necessary to facilitate consistent reporting, to improve possibility of comparing and combining results, to yield high quality evidence and to improve patient care.

\section{Acknowledgements}

We thank Donna Watson (University of Edinburgh) for librarian advice for systematic review search strategy.

\section{Disclosure}


HWL, ELT and MB have no conflicts of interest.

\section{Author contributions}

HWL and MB contributed to conception and design of the study protocol. HWL and ELT extracted data and analysed the results. HWL, ELT and MB drafted and revised the manuscript. All authors gave final approval for the manuscript to be published.

\section{Ethical approval}

Not required

\section{Funding}

None

\section{References}

1. Blustein J, Liu J. Time to consider the risks of caesarean delivery for long term child health. BMJ : British Medical Journal. 2015;350:h2410.

2. Keag OE, Norman JE, Stock SJ. Long-term risks and benefits associated with cesarean delivery for mother, baby, and subsequent pregnancies: Systematic review and meta-analysis. PLOS Medicine. 2018;15(1):e1002494.

3. Betran AP, Torloni MR, Zhang JJ, Gulmezoglu AM. WHO Statement on Caesarean Section Rates. BJOG : an international journal of obstetrics and gynaecology. 2016;123(5):667-70.

4. Lavender T, Hofmeyr GJ, Neilson JP, Kingdon C, Gyte GML. Caesarean section for non-medical reasons at term. Cochrane Database of Systematic Reviews. 2012(3).

5. Macefield RC, Boulind CE, Blazeby JM. Selecting and measuring optimal outcomes for randomised controlled trials in surgery. Langenbecks Arch Surg. 2014;399(3):263-72.

6. Saini P, Loke YK, Gamble C, Altman DG, Williamson PR, Kirkham JJ. Selective reporting bias of harm outcomes within studies: findings from a cohort of systematic reviews. Bmj. 2014;349:g6501.

7. Williamson PR, Altman DG, Blazeby JM, Clarke M, Devane D, Gargon E, et al. Developing core outcome sets for clinical trials: issues to consider. Trials. 2012;13:132.

8. Smith P, Cooper N, Dhillon-Smith R, O'Toole E, Clark TJ, Coomarasamy A. Core Outcome Sets in Miscarriage Trials (COSMisT) study: a study protocol. BMJ Open. 2017;7(11):e018535.

9. Williamson P, Clarke M. The COMET (Core Outcome Measures in Effectiveness Trials) Initiative: its role in improving Cochrane Reviews. Cochrane Database of Systematic Reviews. 2012(4).

10. Khan K. The CROWN Initiative: journal editors invite researchers to develop core outcomes in women's health. BJOG: An International Journal of Obstetrics \& Gynaecology. 2014;121(10):1181-2.

11. Molloy EJ, Gale C, Marsh M, Bearer CF, Devane D, Modi N. Developing core outcome set for women's, newborn, and child health: the CROWN Initiative. Pediatric Research. 2018;84(3):316-7.

12. Duffy JM, van 't Hooft J, Gale C, Brown M, Grobman W, Fitzpatrick R, et al. A protocol for developing, disseminating, and implementing a core outcome set for pre-eclampsia. Pregnancy Hypertens. $2016 ; 6(4): 274-8$.

13. Liberati A, Altman DG, Tetzlaff J, Mulrow C, Gøtzsche PC, Ioannidis JPA, et al. The PRISMA statement for reporting systematic reviews and meta-analyses of studies that evaluate healthcare interventions: explanation and elaboration. BMJ. 2009;339:b2700. 
14. van 't Hooft J, Alfirevic Z, Asztalos EV, Biggio JR, Dugoff L, Hoffman M, et al. CROWN initiative and preterm birth prevention: researchers and editors commit to implement core outcome sets. BJOG : an international journal of obstetrics and gynaecology. 2018;125(1):8-11.

15. Perry H, Duffy JMN, Reed K, Baschat A, Deprest J, Hecher K, et al. A core outcome set for the evaluation of treatments for twin-twin transfusion syndrome. Ultrasound in obstetrics \& gynecology : the official journal of the International Society of Ultrasound in Obstetrics and Gynecology. 2018.

16. de Mattos Lourenco TR, Pergialiotis V, Duffy JMN, Durnea C, Elfituri A, Haddad JM, et al. A systematic review on reporting outcomes and outcome measures in trials on synthetic mesh procedures for pelvic organ prolapse: Urgent action is needed to improve quality of research. Neurourol Urodyn. 2019;38(2):509-24.

17. Durnea CM, Pergialiotis V, Duffy JMN, Bergstrom L, Elfituri A, Doumouchtsis SK. A systematic review of outcome and outcome-measure reporting in randomised trials evaluating surgical interventions for anteriorcompartment vaginal prolapse: a call to action to develop a core outcome set. International urogynecology journal. 2018;29(12):1727-45.

18. Harman NL, Bruce IA, Callery P, Tierney S, Sharif MO, O'Brien K, et al. MOMENT-Management of Otitis Media with Effusion in Cleft Palate: protocol for a systematic review of the literature and identification of a core outcome set using a Delphi survey. Trials. 2013;14:70.

19. Cordoba G, Schwartz L, Woloshin S, Bae H, Gøtzsche PC. Definition, reporting, and interpretation of composite outcomes in clinical trials: systematic review. BMJ. 2010;341:c3920.

20. Macharey G, Vaisanen-Tommiska M, Gissler M, Ulander VM, Rahkonen L, Nuutila M, et al. Neurodevelopmental outcome at the age of 4 years according to the planned mode of delivery in term breech presentation: A nationwide, population-based record linkage study. Journal of Perinatal Medicine. 2018;46(3):333-9.

21. Maier B, Georgoulopoulos A, Zajc M, Jaeger T, Zuchna C, Hasenoehrl G. Fetal outcome for infants in breech by method of delivery: Experiences with a stand-by service system of senior obstetricians and women's choices of mode of delivery. Journal of Perinatal Medicine. 2011;39(4):385-90.

22. Robson SJ, de Costa C, Woods C, Ding P, Rane A. Maternal-choice caesarean section versus planned vaginal birth in low-risk primigravid women. Australian and New Zealand Journal of Obstetrics and Gynaecology. 2018;58(4):469-73.

23. Duffy J, Rolph R, Gale C, Hirsch M, Khan K, Ziebland S, et al. Core outcome sets in women's and newborn health: a systematic review. BJOG: An International Journal of Obstetrics \& Gynaecology. 2017;124(10):1481-9.

24. Kirkham JJ, Boers M, Tugwell P, Clarke M, Williamson PR. Outcome measures in rheumatoid arthritis randomised trials over the last 50 years. Trials. 2013;14:324.

25. Chalmers I, Glasziou P. Avoidable waste in the production and reporting of research evidence. Lancet. 2009;374(9683):86-9.

26. Hirsch M, Duffy JMN, Barker C, Hummelshoj L, Johnson NP, Mol B, et al. Protocol for developing, disseminating and implementing a core outcome set for endometriosis. BMJ Open. 2016;6(12):e013998.

27. Webbe J, Brunton G, Ali S, Duffy JM, Modi N, Gale C. Developing, implementing and disseminating a core outcome set for neonatal medicine. BMJ Paediatr Open. 2017;1(1):e000048.

28. Kirkham JJ, Dwan KM, Altman DG, Gamble C, Dodd S, Smyth R, et al. The impact of outcome reporting bias in randomised controlled trials on a cohort of systematic reviews. BMJ. 2010;340:c365.

\section{Hosted file}

Figure.docx available at https://authorea.com/users/324111/articles/452423-reportedoutcomes-for-planned-caesarean-section-versus-planned-vaginal-birth-in-women-aged-18- 
45-a-systematic-review 